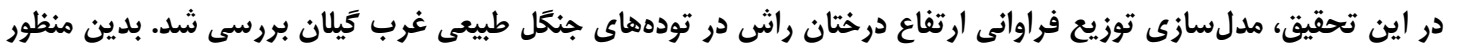

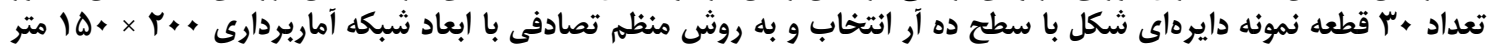

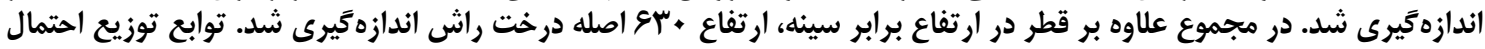

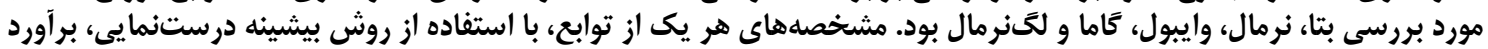

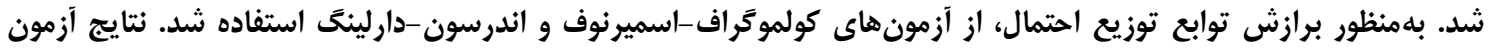

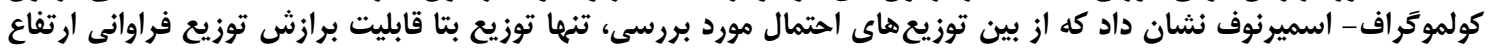

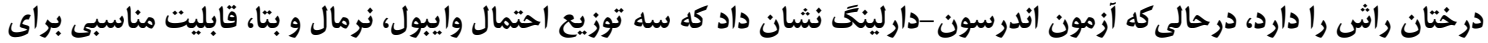

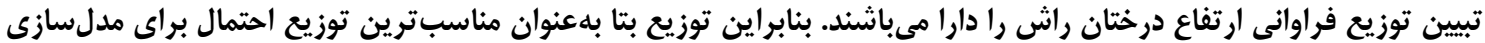
توزيع فراوانى درختان راش در منطقاع درخ مورد مطالعه، انتخاب شدان

وازههاى كليدى: ارتفاع، تودههاى طبيعى، راش، توزيع احتمال، ماسال

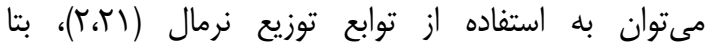

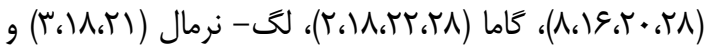

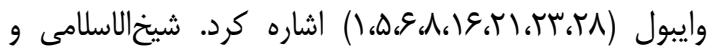

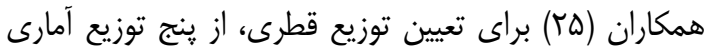

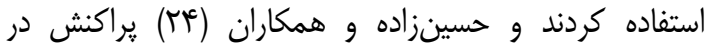

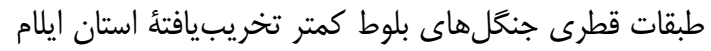

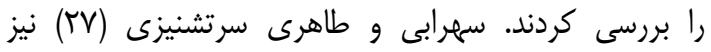

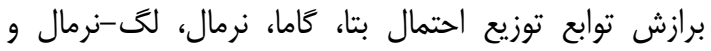

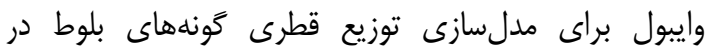

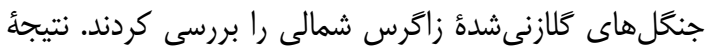

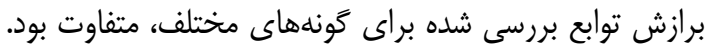

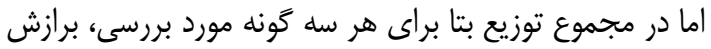

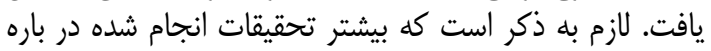

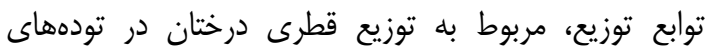

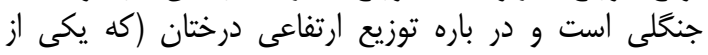

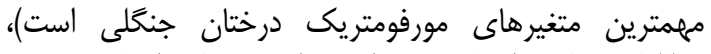

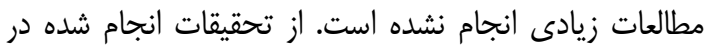

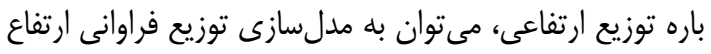

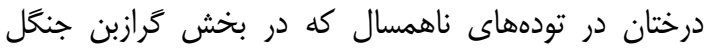

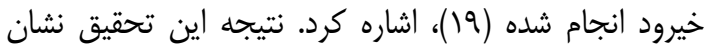

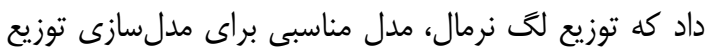

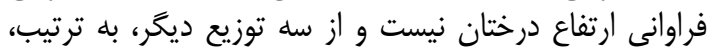

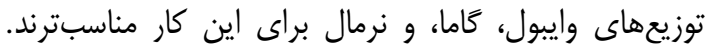

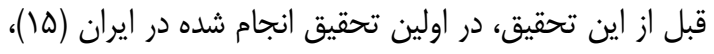

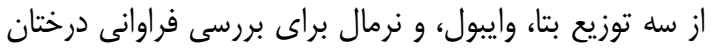

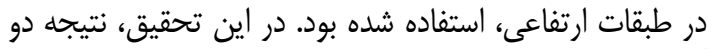

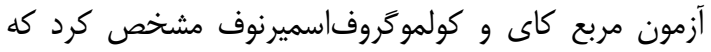
هيج يك از توزيعهاى فوق، مدل مناسبى براى اين منظور كنري

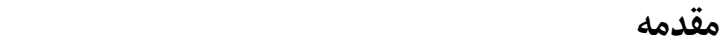

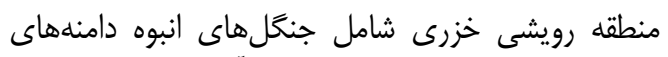

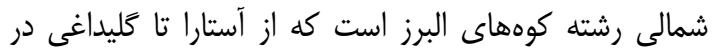

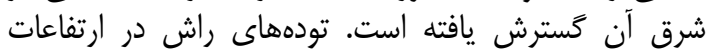

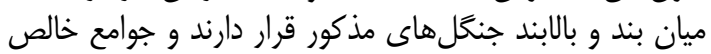

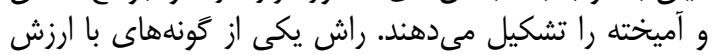

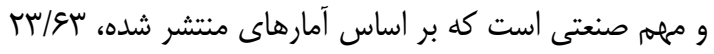

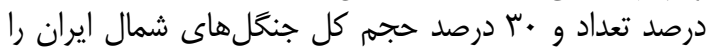

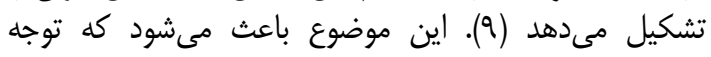

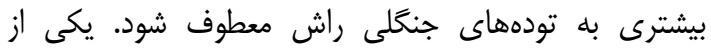

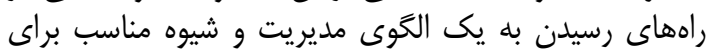

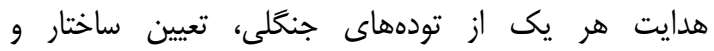

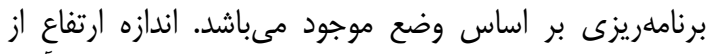

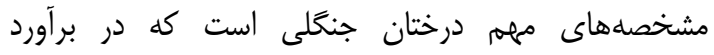

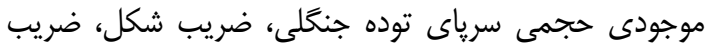

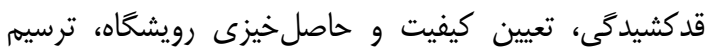

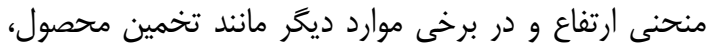

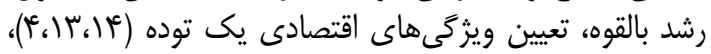

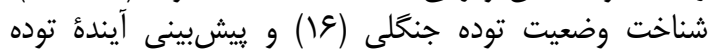

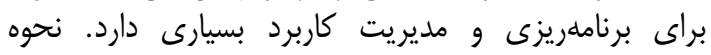

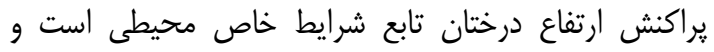

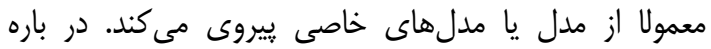

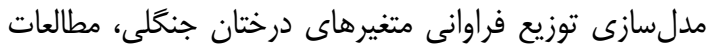

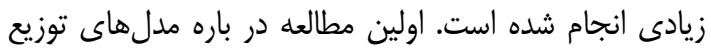

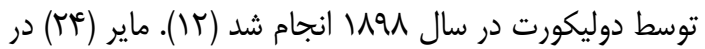

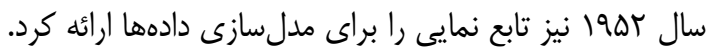

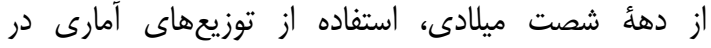
يزوهشهاى جنكّل متداولتر شد (•r). از مطالعات انجام شده 


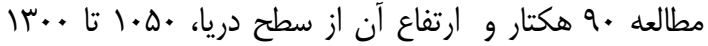

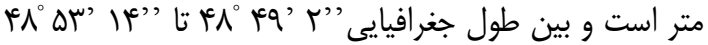

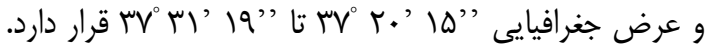

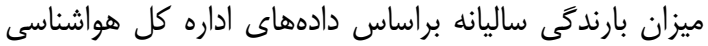

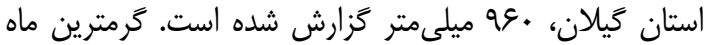

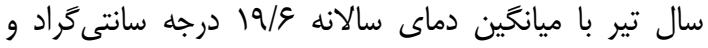

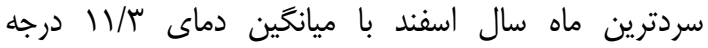

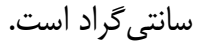

روش جمعآورى اطلاعات، محاسبات اوليه و انتخاب

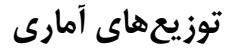

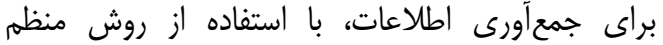

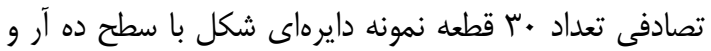

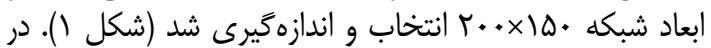

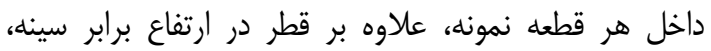

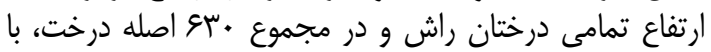

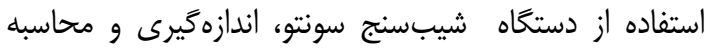

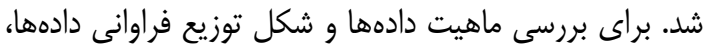

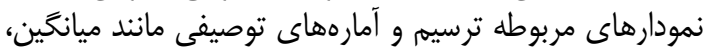

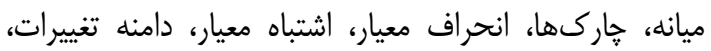

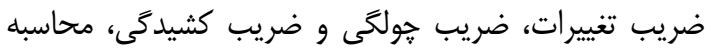

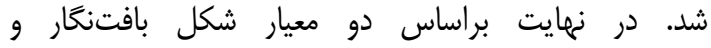

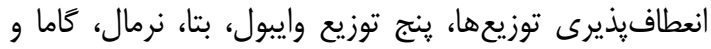

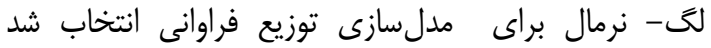

.(1.. (19)

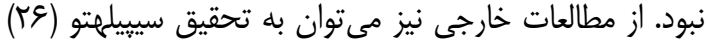

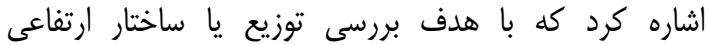

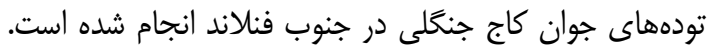

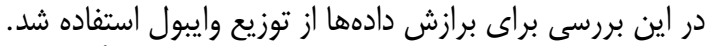

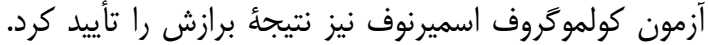

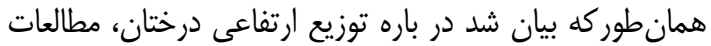

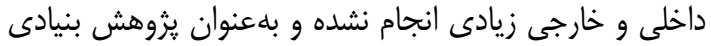

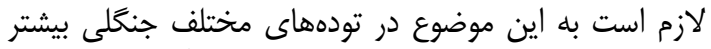

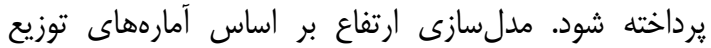

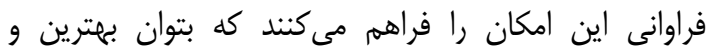

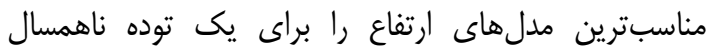

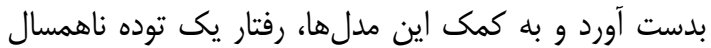

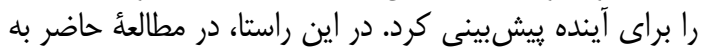

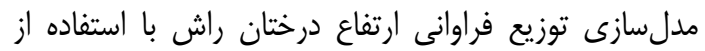

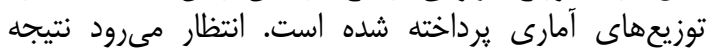

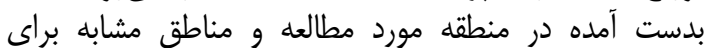
اهداف و كاربردهاى ذكر شده، بكار كرفته شود.

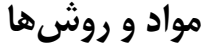 منطقه مورد مطالعه}

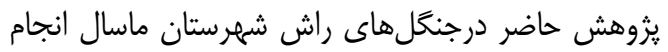

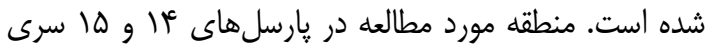

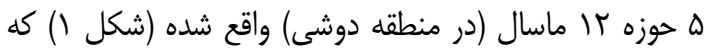

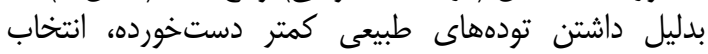

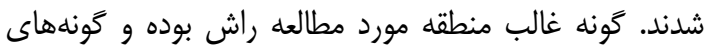
همراه آن ممرز، توسكا و شيردار است. مساحت منطقه مورد ماند
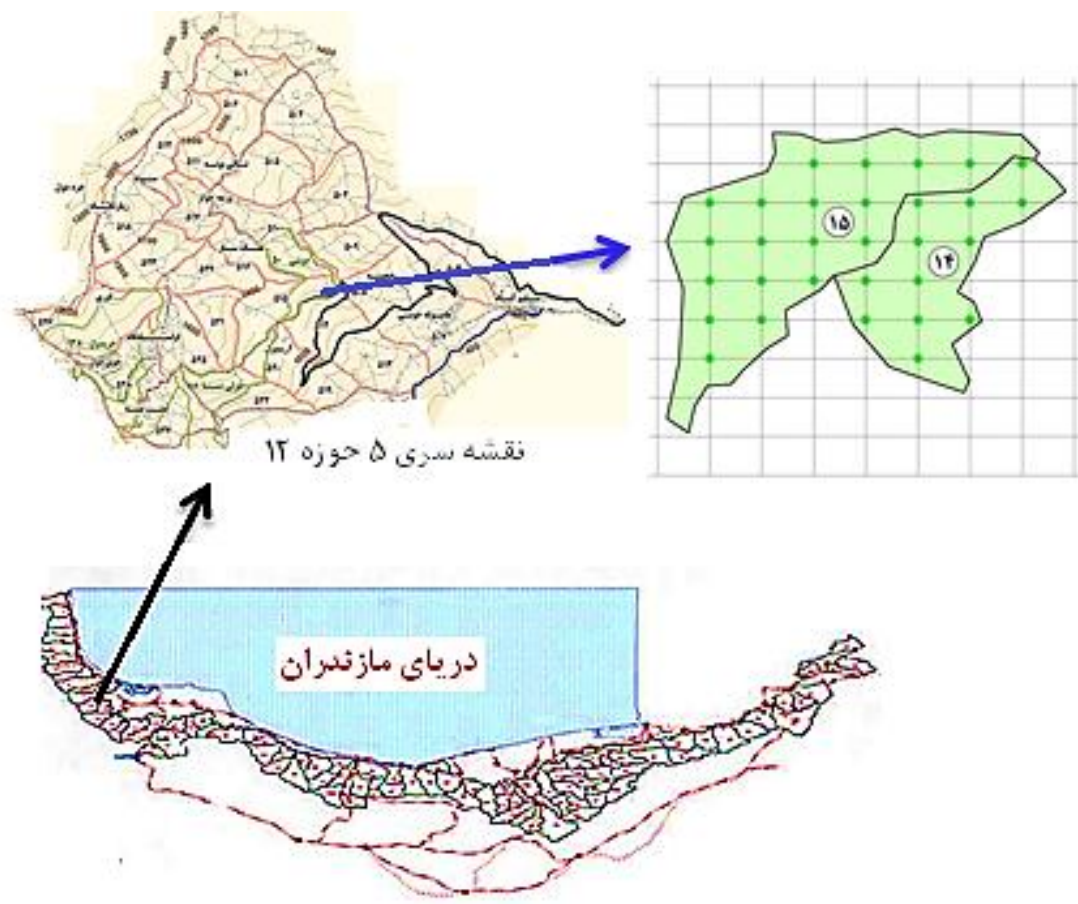

شكل ا- موقعيت منطقه مورد مطالعه به همراه شبكه آماربردارى بر روى يارسل هاى مورد بررسى

Figure 1. The location of the study area along with the sampling network on the studied parcells 


$$
f(X)=\frac{(x-a)^{a_{1-1}}(b-x)^{a_{2-1}}}{B\left(\sigma_{1}, \sigma_{2}\right)(b-a)^{a_{1}+a_{2-1}}}
$$

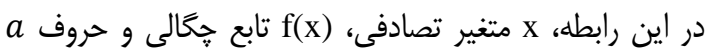
و bشخصdهاى كرانهاى و حروف a شكل است. برازش توزيعها و سنجش نكويى برازش

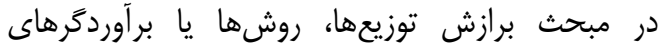

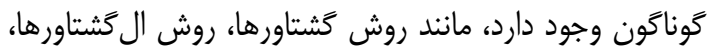

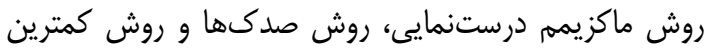

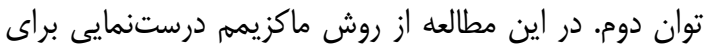

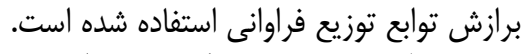

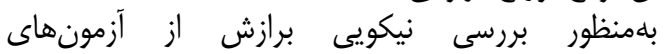

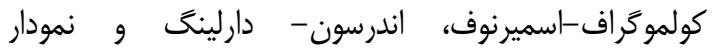

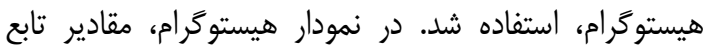

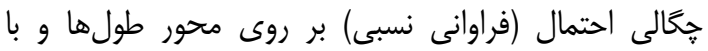

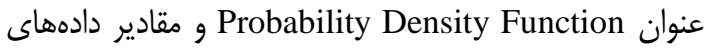

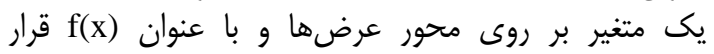

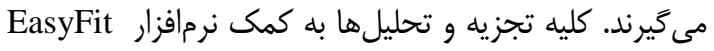
5.5 انجام شد.

نتايج بدست آمده از محاسبات اوليه بر رودى دارد دادههاى

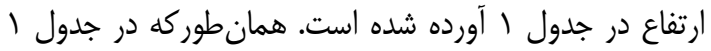

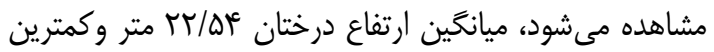

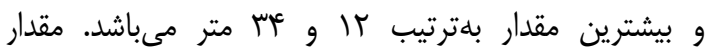

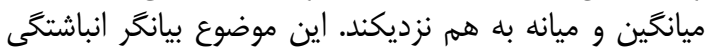

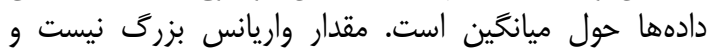

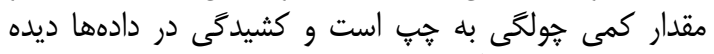

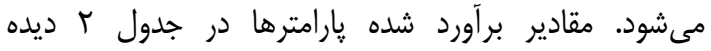

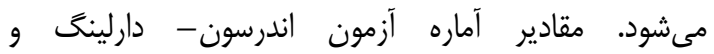

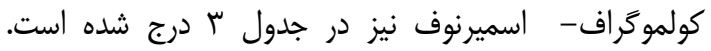

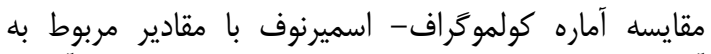

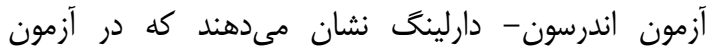

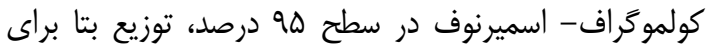

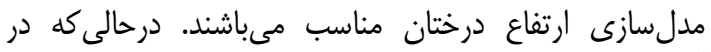

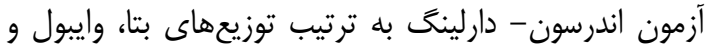

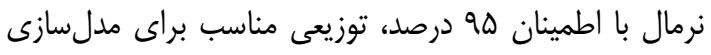

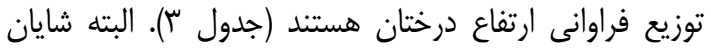

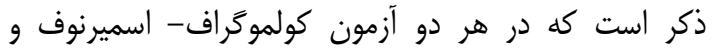

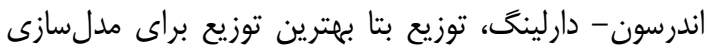

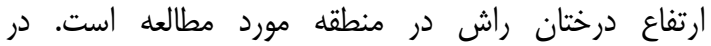

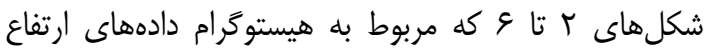

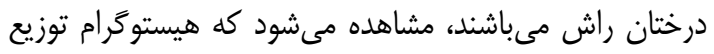

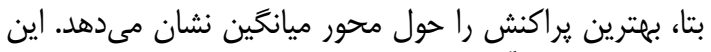

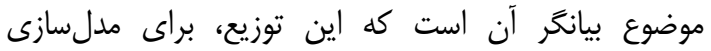

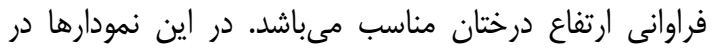

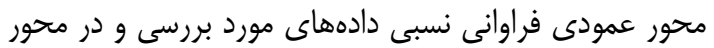

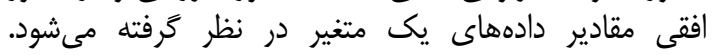

توزيع وايبول

توزيعى ييوسته است كه انعطافيذيرى بالايى داردي دارد. اين

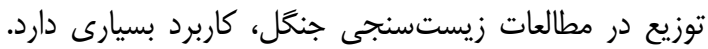

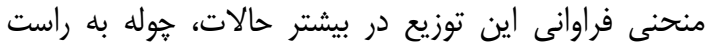
است. تابع جّالى اين توزيع به قرار زير است (رابطه ()): $\mathrm{f}(\mathrm{X})=\frac{\alpha}{\beta^{\alpha}} \mathrm{X}^{\alpha-1} \mathrm{e}^{-\left(\frac{\mathrm{X}}{\bar{\beta}}\right)^{\alpha}}$

$0 \leq x<+\infty$

$$
\alpha, \beta>0
$$

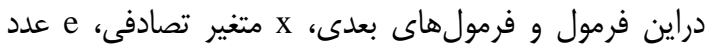

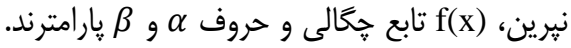

توزيع تَاما

توزيعى ييوسته است كه انعطاف يذيرى خوبى خافى دارد. منحنى إنى

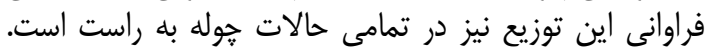

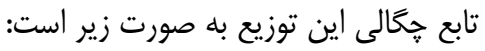

$$
f(X)=\frac{x}{\beta^{\alpha} \tau(\alpha)} e^{\left(-\frac{x}{\beta}\right)} 0 \leq x<+\infty \quad: \quad \text { رابطه }
$$

در اين رابطه، X متغير تصادفى، e عدد نير، f(x) تابع קֶَالى و

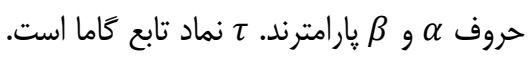
توزيع نرمال را مىتوان اساسىترين توزيع در آماركلاسيك دانست، زيرا پايه بسيارى از روشهاى آمار كلاسيك بر اين توزيع كم داسي و تابع جَالى آن به صورت زير است (رابطه بَّ): $f(x)=\frac{1}{\sigma \sqrt{2 \pi}} e^{\wedge}\left[-\left(x-\mu^{\wedge} 2 / 2 \sigma^{2}\right] \quad\right.$ رابطه (r) $-\infty \leq x \quad \mu<+\infty \quad \sigma>0$

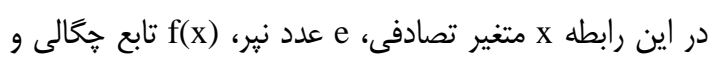

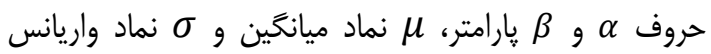

\section{توزيع لَى نرمال}

نيز توزيعى ييوسته است. اگر متغيرى تصادل تصادفى، توزيع

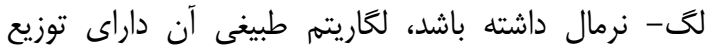

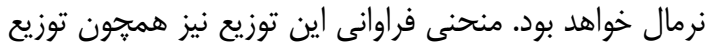

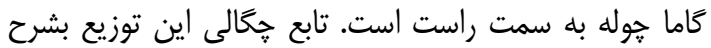
زير است (رابطه أ):
$\mathrm{f}(\mathrm{x})=\frac{1}{\sigma \sqrt{2 \pi}} \mathrm{e}^{\wedge}\left[-\left(\ln \mathrm{x}-\mu^{\wedge} 2 / 2 \sigma^{2}\right]\right.$
رابطه (ז):

$\mathrm{x}, \mu, \sigma>0$

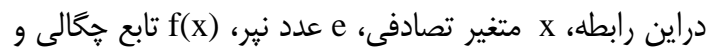

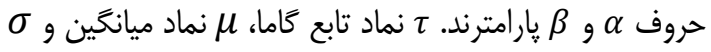
نماد واريانس است. إن نماد لخاريتم طبيعى است. توزيع بتا تيماد

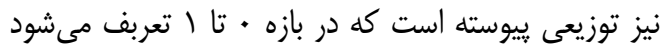

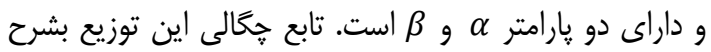
زير است (رابطه ه): 


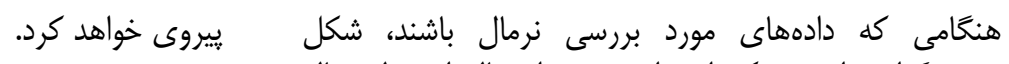
هيستوكرام براى هريك از توابع توزيع احتمال از خط نرمال

Table 1. Descriptive statistics of tree's height $(\mathrm{m})$

\begin{tabular}{|c|c|c|c|}
\hline مقدار & أماره & مقدار & أماره \\
\hline$\pi$ & كمينه & $T r / \Delta F$ & ميانگين \\
\hline$r \cdot$ & جارى اول & . & اشتباه معيار \\
\hline r & ميانه & $\mid V / \Gamma \Lambda$ & واريانس \\
\hline$r \Delta$ & جارك سوم & $\cdot / \Lambda \Lambda \Delta$ & ضريب تغيرات \\
\hline re & بيشينه & $-\cdot / \Delta F \Delta$ & ضريب جولىى \\
\hline r & دامنه تغييرات & $\cdot / \mathrm{VA}$ & ضريب كشيدگى \\
\hline
\end{tabular}

Table 2. Parameters of probability distribution

جدول r- مقادير يارامترهاى برآوردشده توزيعها

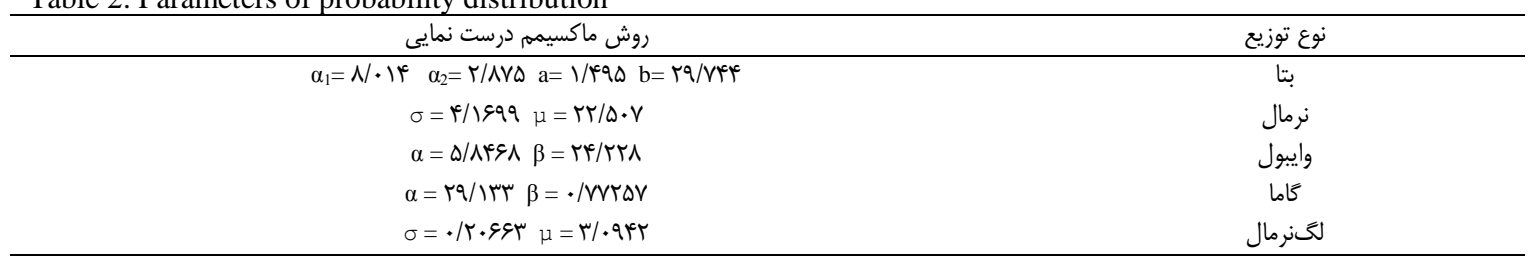

جدول ب- مقادير آماره آزمونهاى كولموكراف-اسميرنوف و اندرسون -دارلينگ براى توزيعها Table 3. Statistical value of the Kolmogorov-Smirnov and Anderson-Darling tests for distributions

\begin{tabular}{|c|c|c|c|c|c|c|}
\hline \multirow[b]{2}{*}{ سطح معنىدارى } & \multicolumn{2}{|c|}{ اندرسون -دارلينگ } & \multicolumn{2}{|c|}{ كولموگراف-اسميرنوف } & \multirow[b]{2}{*}{ أماره } & \multirow[t]{2}{*}{ توزيع } \\
\hline & رتبه & أماره & سطح معنى دارى & رتبه & & \\
\hline$N s$ & 1 & I/\&DAr & $N s$ & 1 & $\cdot / 1 \cdots$ & بتا \\
\hline Ns & r & r/r & $*$ & r & س אוII. & وايبول \\
\hline$*$ & r & $r / \varepsilon q$ & * & f & . METE & كاما \\
\hline$*$ & $\Delta$ & f/Arrl & * & $\Delta$ & $\cdot|| \Delta \wedge \mid$ & ل لى نرمال \\
\hline
\end{tabular}

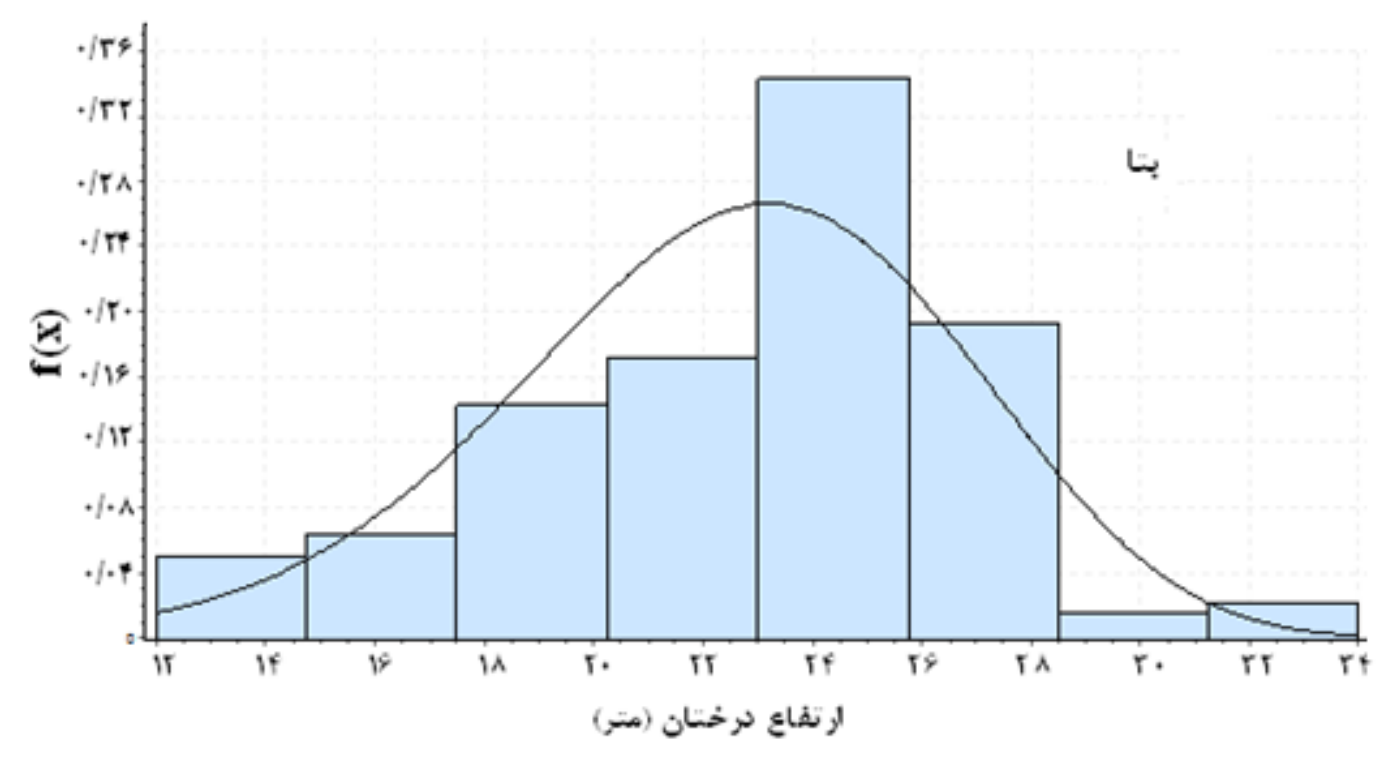

شكل r- هيستو

Figure 2. Histogram of the beta distribution 


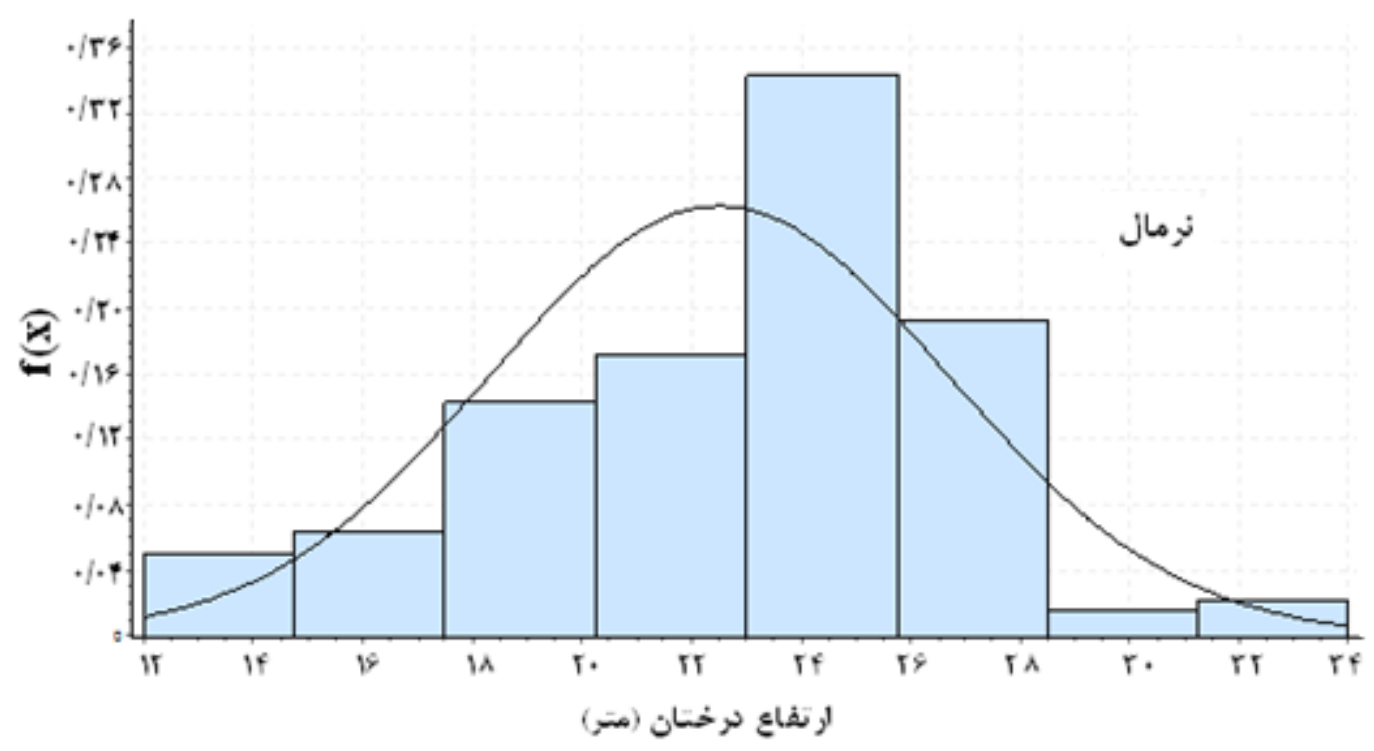

شكل س- هيستو كرام توزيع نرمال

Figure 3. Histogram of the normal distribution

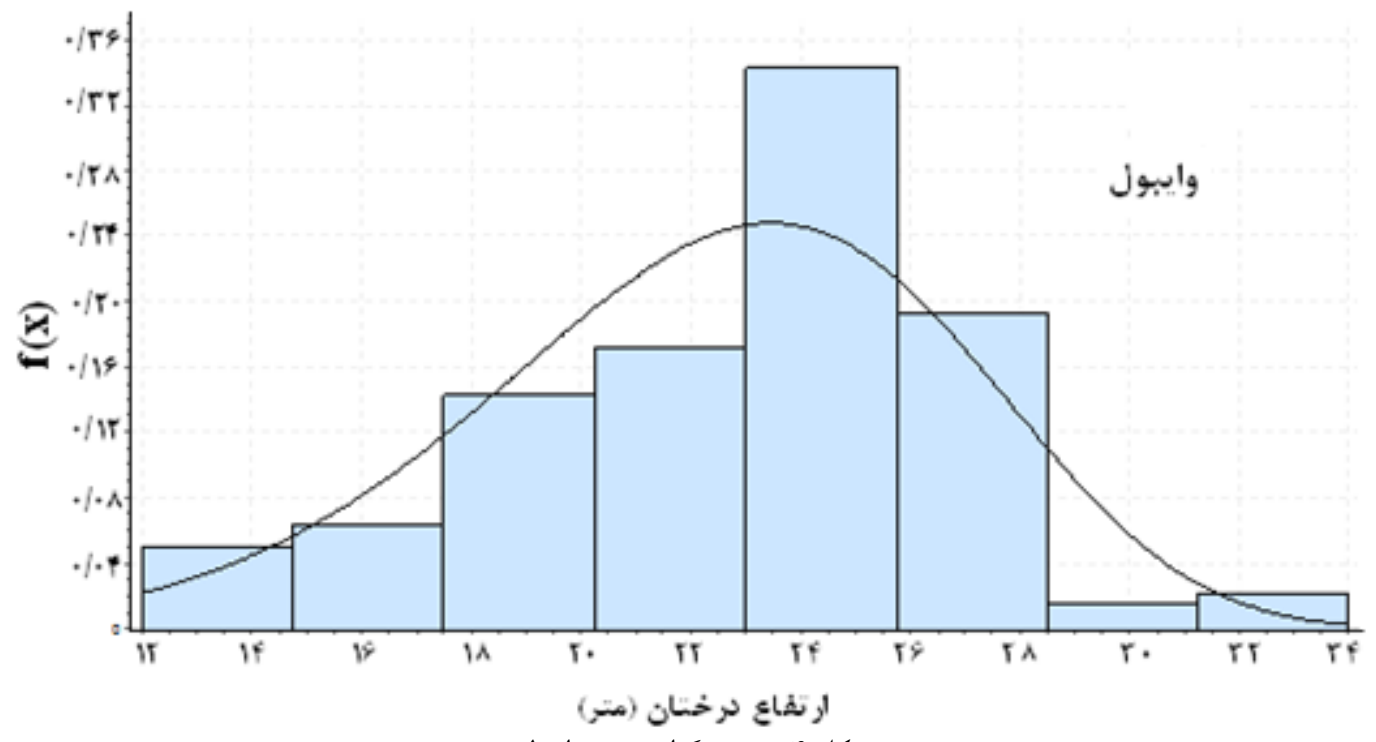

شكل ع - هيستو

Figure 4. Histogram of the weibull distribution 


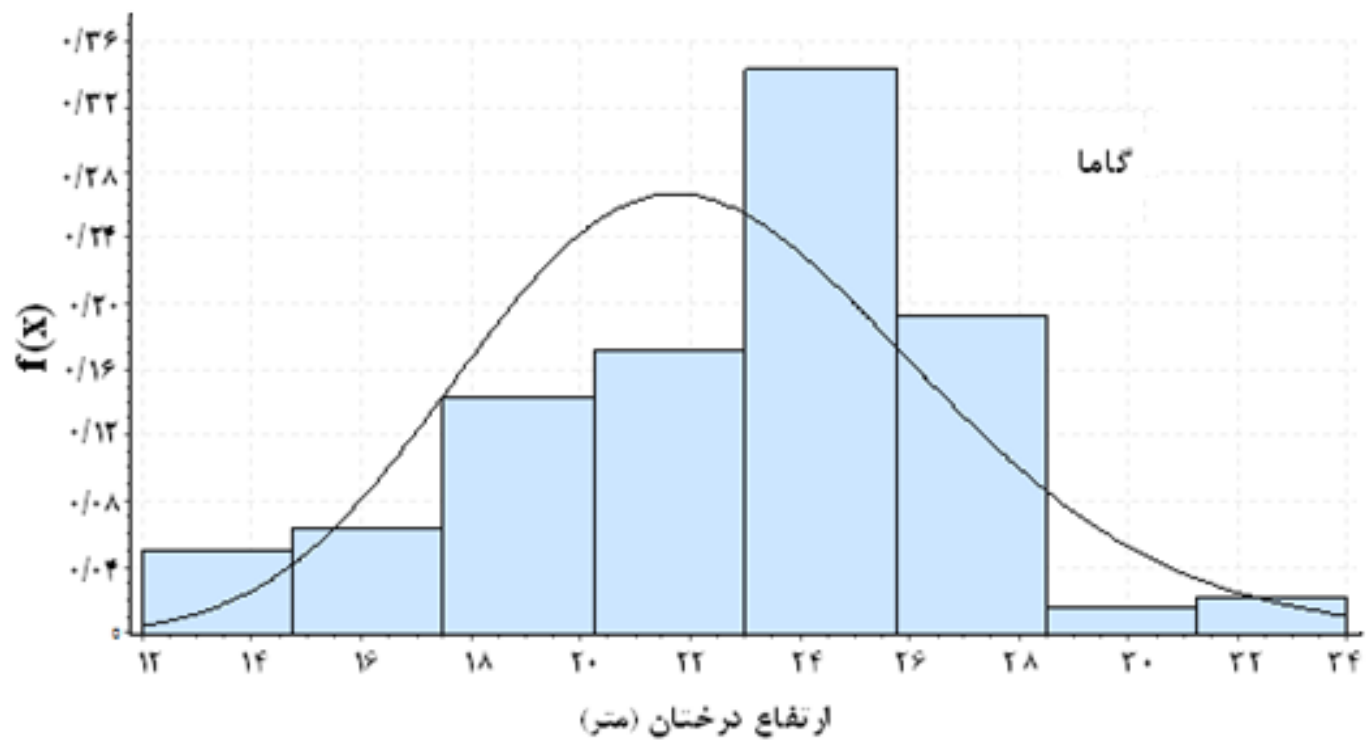

شكل ه- هيستو كرام توزيع كاما

Figure 5. Histogram of the gamma distribution

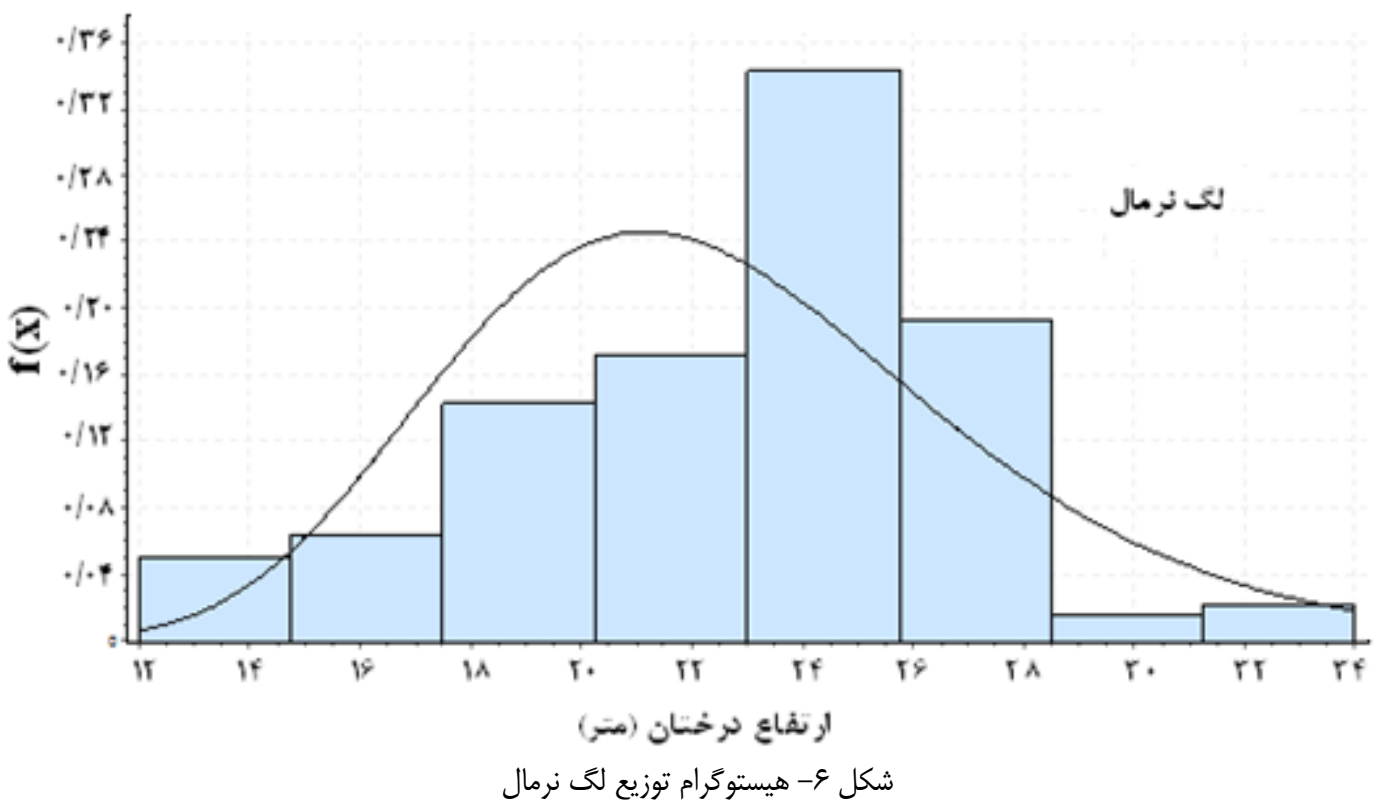

Figure 6. Histogram of the lognormal distribution

بيانكر انباشتخى دادهها حول ميانكين است. مقادير بدست

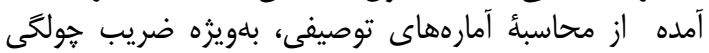

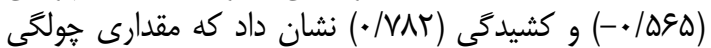

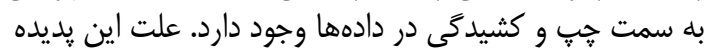

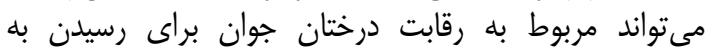

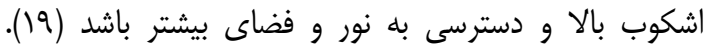

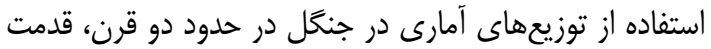

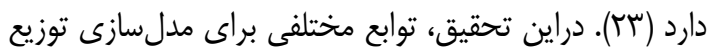
فراوانى ارتفاع درختان راش درجنكلى توايع مخاى ماسال (كيلان)
مدلهاى رياضى ابزارهاى كارآمدى براى مدلسازى مدي

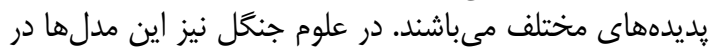

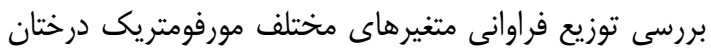

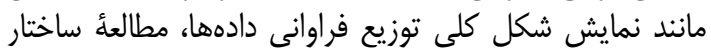

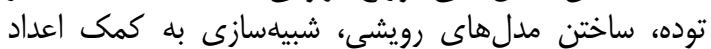

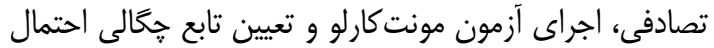

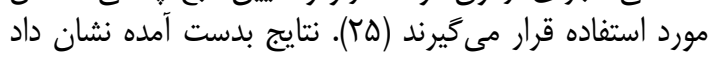

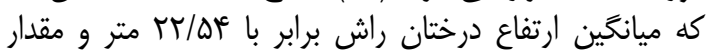
ميانه برابر با بr متر بود. مشابهت مقان مقدار ميانكين و ميانه، 
بررسى نشده است. همجنين نتيجه تحقيق سييبلهتو (عآ) با

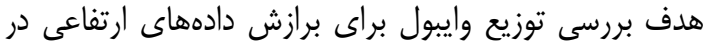

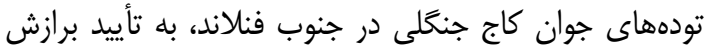

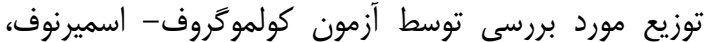

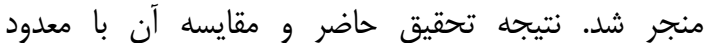

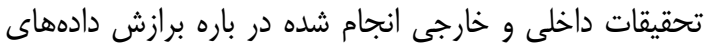

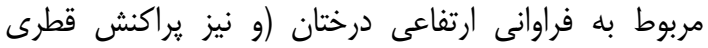

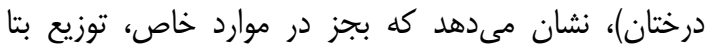

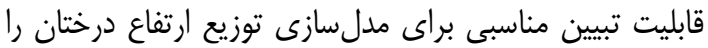

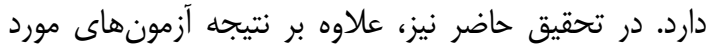

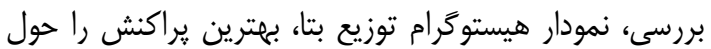

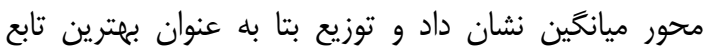

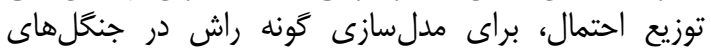
كيلان (ماسال)، بدست آمد. نتيجها

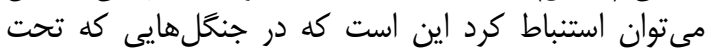

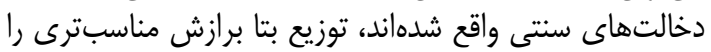

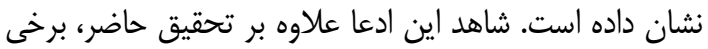

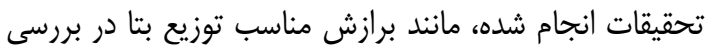

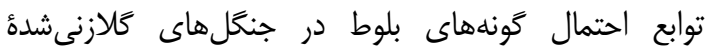

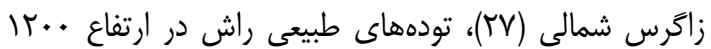

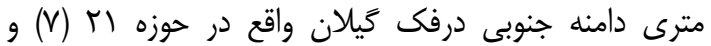

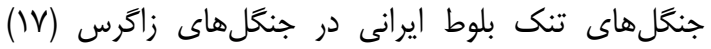
مىباشد. ييشنهاد مى شود كه در باري باره اين موضوع در در تحقيقات آتى، بررسى هاى بيشترى انجام شود.
بررسى شد. مقايسه اين توابع نشان داد كه تابع توزيع بتا،

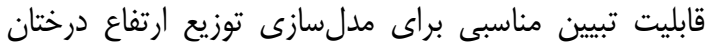

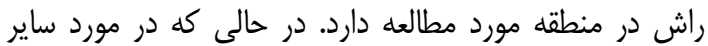

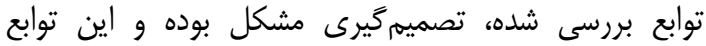

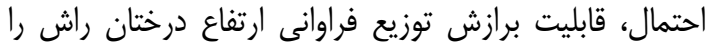

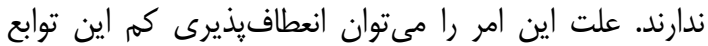

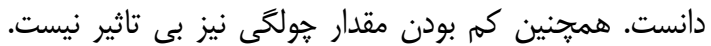

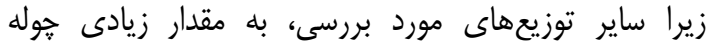

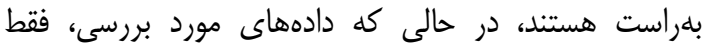

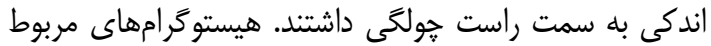

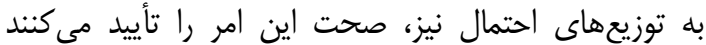

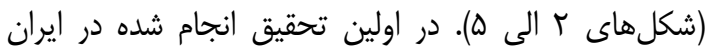

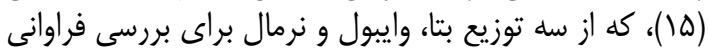

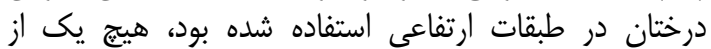

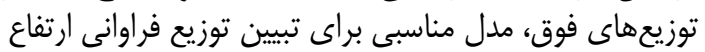

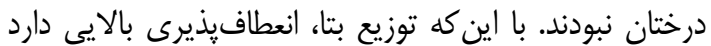

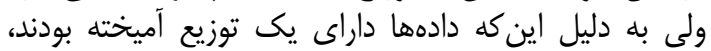

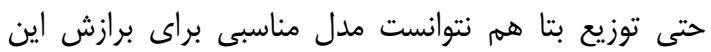

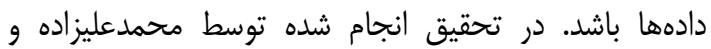

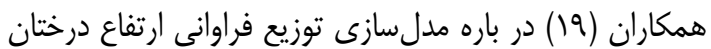

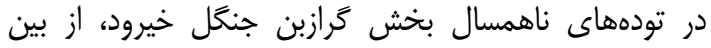

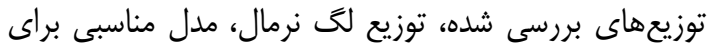

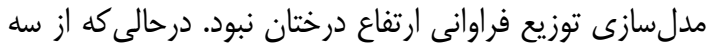

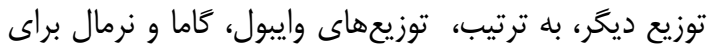
اين كار مناسبتر بودند. در اين تحقيق درئ در باره توزيع بتا

1. Bailey, R.L. and T.R. Dell. 1973. Quantifying diameter distributions with the Weibull function, Forest Science, 19: 97-104.

2. Bailey, L.R. 1980. Individual tree growth derived from diameter distribution models, Forest Science, 26: 626-632.

3. Bliss, C.I. and K.A. Reinker. 1964. A lognormal approach to diameter distributions in even-aged stands, Forest Science, 10: 350-360.

4. Brooks, J.R. and H.V. Wiant. 2004. A simple technique for estimating cubic foot volume yields, Forest Ecology and Management, 203: 373-380.

5. Bullock, B.P. and E.L. Boone. 2007. Deriving tree diameter distributions using Bayesian model averaging, Forest Ecology and Management, 242: 127-132.

6. Cao, Q.V. 2004. Predicting parameters of a Weibull function for modeling diameter distribution. Forest Science, 50: 682-685.

7. Eslami, A.R., B. Karimi, H. Payam and O.K. Derakhshan. 2011. Investigation of the structure and distribution diameter classes models in beech forests of Northern Iran, African Journal of Agricultural Research, 6: 2157-2165.

8. Fallah, A., M. Zobeiri and M.R. Marvie Mohajer. 2006. An appropriate model for distribution of diameter classes of Natural Beech Stands in the Sangdeh \& Shastkolateh Forests, Iranian Journal of Forest, 58: 813-821 (In Persian).

9. FAO. 2001. Global forest resources assessment 2000. Main Report. FAO Forestry Paper 140. Rome, $479 \mathrm{pp}$.

10. Hoorfar, A. 2007. Engineering statistics, course for Ph.D. students of irrigation, Faculty of Agricultural Engineering and Technology, University of Tehran, $120 \mathrm{pp}$ (In Persian).

11. Hosseinzadeh, J., M. Namiranian, M.R. Marvi Mohajer and Gh. Zahedi Amiri. 2004. Structure of Less Degraded Oak Forests in Illam Province (Southwest Iran), Iranian Journal of Natural Resources, 57: 75-90 (In Persian).

12. Johnson, E. 2000. Forest Sampling Desk Reference, CRC Press LLC, 985 pp.

13. Kangas, A.S. 1998. Uncertainty in growth and yield projections due to annual variation of diameter growth, Forest Ecology and Management, 108: 223-230. 
14. Knobel, B.R. and H.E. Burkhart. 1991. A bivariate distribution approach to modeling forest diameter distributions at two points in time, Biometrics, 47: 241-253.

15. Mataji, A. 1999. Study on tree distribution in height classes in natural forests. M.Sc. thesis in forestry, Faculty of Natural Recourses, University of Tehran, 76 pp (In Persian).

16. Mattaji, A., S.M. Hojjati and M. Namiranian. 2000. A study of tree distribution in diameter classes in natural forests using probability distributions. Iranian Journal of Natural Resources, 53: 165-173 (In Persian).

17. Mirzaei, M., J. Aziz, A. Mahdavi and A. Mohammad Rad. 2016. Modeling frequency distributions of tree height, diameter and crown area by six probability functions for open forests of Quercus persica in Iran, Journal of Forestry Research, 27: 901-906.

18. Mohammadalizadeh, Kh., M. Zobeiri, M. Namiranian, A. Hoorfar and M.R. Marvie Mohajer. 2009. Fitting of diameter distribution using some statistical models (distributions), Iranian Journal of Forest and Poplar Research, 17: 116-124 (In Persian).

19. Mohammadalizadeh, Kh., M. Zobeiri, M. Namiranian, A. Hoorfar and M.R. Marvie Mohajer. 2013. Modeling of height distribution of trees in uneven aged forest stands (Case Study: Gorazbon district of Kheyroud forest), Iranian Journal of Forest and Wood Product (Iranian Journal of Natural Resources), 66: 155-165 (In Persian).

20. Namiranian, M. 1990. Application of probability models in description of distribution of trees in diameter classes. Iranian Journal of Natural Resources, 44: 93-108 (In Persian).

21. Nanang, D.M. 1998. Suitability of the normal, lognormal and Weibull distributions for fitting diameter distributions of neem plantations in northern Ghana, Forest Ecology and Management, 103: 1-7.

22. Nelson, T.C. 1964. Diameter distribution and growth of loblolly pine, Forest Science, 10: 105-115.

23. Nord-Larson, T. and Q.V. Cao. 2006. A diameter distribution model for even-aged beech in Denmark. Forest Ecology and Management, 231: 218-225.

24. Rubin, B.D., P.D. Manion and D.F. Langendoen. 2006. Diameter distributions and structural sustainability in forests, Forest Ecology and Management, 222: 427-438.

25. Sheykholeslami, A., KH. Kia-Pasha and A. Kia-Lashaki. 2011. A study of tree distribution in diameter classes in natural forests of Iran. Annals of Biological Research, 2: 283- 290.

26. Siipilehto, J. 2006. Height distribution of Scots pine sapling stands affected by retained tree and edge stand competition. Silva Fennica, 40: 473-486.

27. Sohrabi, H. and M.J. Taheri Sarteshnizi. 2012. Fitting probability distribution functions for modeling diameter distribution of oak species in pollarded northern Zagros forests (Case study: ArmardehBaneh), Iranian Journal of Forest, 4: 333-343 (In Persian).

28. Zheng, L. and X. Zhou. 2010. Diameter distribution of trees in natural stands managed on polycyclic cutting system. Forest studies in China, 12: 21-25.

29. Zwillinger, D. and S. Kokoska. 2000. CRC Standard probability and statistics table and formulae. Chapman \& Hall/CRC, 554 pp. 


\title{
Fitting Tree Height Distributions in Natural Beech Forest Stands of Guilan (Case Study: Masal)
}

\section{Iraj Hassanzad Navroodi ${ }^{1}$ and Ismaeil Moradi Emam Qeysi ${ }^{2}$}

1- Associate Professor, University of Guilan (Corresponding author: iraj.hassanzad@ gmail.com)

2- Graduated M.Sc. Student, University of Guilan

Received: January 7, 2017 Accepted: April 10, 2017

\begin{abstract}
In this research, modeling tree height distributions of beech in natural forests of Masal located in Guilan province, was investigated. Inventory was carried out using systematic random sampling with network dimensions of $150 \times 200 \mathrm{~m}$ and area sample plot of 0.1 ha. DBH and heights of 630 beech trees in 30 sample plots were measured. Beta, Gamma, Normal, Lognormal and Weibull probability distribution function were surveyed. Characteristics of distribution functions were estimated by use of maximum likelihood method. Actual probability and probability derived from functions was compared using Kolmogorov-Smirnov and Anderson-Darling tests. Results of Kolmogorov-Smirnov test showed that only Beta probability distribution function was suitable for modeling height distribution of beech; while results of Anderson-Darling tests demonstrated probability distribution functions of Beta, Normal, and Weibull were fitted to the height distribution of trees. Therefore probability distribution function of Beta was selected for modeling height distribution of beech.
\end{abstract}

Keywords: Beech, Height, Natural forest stands, Masal, Probability distribution 